\title{
Mucoepidermoid Carcinoma in Carcinosarcoma of the Lung - A Rare Combination
}

\author{
Meetu AGRAWAL, Tara Roshni PAUL, Shantveer G. UPPIN, Sundaram CHALLA \\ Department of Pathology, Nizam l's Institute of Medical Sciences, HYDERABAD, INDIA
}

\section{ABSTRACT}

Carcinosarcomas of the lung are uncommon neoplasms and are presently categorized along with sarcomatoid carcinomas in the WHO 2004 classification. Morphological recognition of individual components in a surgical specimen is usually straightforward. The respiratory epithelium is also a rare site for salivary gland type tumors. A 72-year-old lady who was investigated for an episode of hemoptysis was found to have a well circumscribed intraluminal tumor. The histo-morphological features were unique and composed of mucoepidermoid carcinoma and undifferentiated sarcoma. To the best of our knowledge, this unusual morphological combination has never been reported in literature.

Key Words: Lung, Carcinosarcoma, Mucoepidermoid carcinoma

\section{INTRODUCTION}

Carcinosarcoma is defined as a tumor composed of epithelial and mesenchymal elements, both components being malignant (WHO 2004) $(1,2)$. Though carcinosarcoma has been described at all sites where a carcinoma can occur (uterus, breast, thyroid, esophagus, lung), it is overall a rare diagnosis. In the lung, it accounts for less than $0.3 \%$ of all lung cancers and has been grouped along with sarcomatoid carcinomas (3). Primary salivary gland type lung tumors are a distinct rare category of lung malignancy with well-defined characteristics $(4,5)$. Mucoepidermoid carcinoma forms the second commonest type (after adenoid cystic carcinoma) in this category. However, it has never been reported as a component of carcinosarcoma, where conventional nonsmall cell lung cancers are commoner. This case is reported here because of an uncommon morphological diagnosis.

\section{CASE REPORT}

A 72-year-old lady presented with a single episode of hemoptysis 15 days back. There had been no prior episodes, fever, breathlessness, cough, expectoration, chest pain or loss of weight. General examination was unremarkable. Respiratory rate was 14/minute. Ultrasound abdomen showed all viscera in their normal anatomical condition. Chest radiograph and CT scan revealed a single intrabronchial mixed density mass in the right upper bronchus with no evidence of atelectasis. FNA done outside this hospital was suggestive of a "malignant spindle cell tumor". Evaluation for any primary tumor elsewhere and workup

(Turk Patoloji Derg 2016, 32:122-125)

Received : 17.03.2013 Accepted : 14.04.2013 for metastasis was negative. She underwent right upper and middle lobectomy along with hilar lymph node clearance for this lung tumor.

Surgical specimen: The right upper main bronchus was dilated with an intraluminal growth measuring $4.8 \times 3 \times 1.2 \mathrm{~cm}$. Cut surface was variegated with hemorrhagic areas and patchy consolidation in the distal lung (Figure 1A). Mapped sections from the specimen submitted showed the lesion composed of two discreet areas with different morphologies. There was an epithelial component composed of a variable admixture of nests and solid islands of squamous, intermediate and mucin producing cells (Figure 1B-D). The acidic mucin was stained positively by Alcian PAS stain. The mesenchymal component was composed of short and long interlacing fascicles and focal sheet like arrangement of spindle shaped cells with irregularly interspersed giant cells. The cells had moderately pleomorphic vesicular nuclei, conspicuous nucleoli and scant to moderate cytoplasm (Figure 1E). Interspersed tumor giant cells were seen. Mitosis was 4-5/10HPF. Areas of hemorrhage were seen but no osseous or cartilaginous component was noted. Repeated deeper sections and extensive grossing till exhaustion of the tumor did not yield any differentiation of the sarcomatous component.

The remaining lung parenchyma showed focal organized fibrinous exudates with mononuclear inflammatory cells. Hilar lymph nodes were negative for metastasis. Immunohistochemistry was done with a panel comprising

Correspondence: Tara Roshni PAUL

Nizam's Institute of Medical Sciences, Department of Pathology,

HYDERABAD, INDIA

E-mail: troshnip@yahoo.co.in Phone: +91 0402899786 
of vimentin, pancytokeratin (CK), Ki67 and thyroid transcription factor (TTF-1). CK was positive in epithelial and vimentin was strongly expressed in the mesenchymal component. Ki67 in the mesenchymal component was $28 \%$, thus confirming its sarcomatous nature (Figure 2 A-C). TTF-1 was positive in pulmonary epithelial cells but negative in the neoplastic component. Desmin, myogenin, S-100, smooth muscle actin, CD34 were negative.

A diagnosis of pulmonary carcinosarcoma with undifferentiated sarcoma was given. No adjuvant therapy was given as the tumor was entirely intra-bronchial and there was no evidence of metastasis elsewhere. The patient is asymptomatic after 7 months of follow-up.

\section{DISCUSSION}

Carcinosarcomas are rare tumors accounting for less than $0.3 \%$ of all lung malignancies (4). The commonest carcinomatous component is a squamous cell carcinoma and sarcomatous component is usually an osteosarcoma, chondrosarcoma or a rhabdomyosarcoma (6). Also, salivary gland tumors in the lung have a histologic resemblance to their glandular counterparts and are well described but rare (5). This peculiar case is probably the first of its kind where a mucoepidermoid carcinoma was part of a carcinosarcoma. Despite extensive sampling, we could not characterize the sarcoma into any morphological category and it remained an undifferentiated sarcoma.
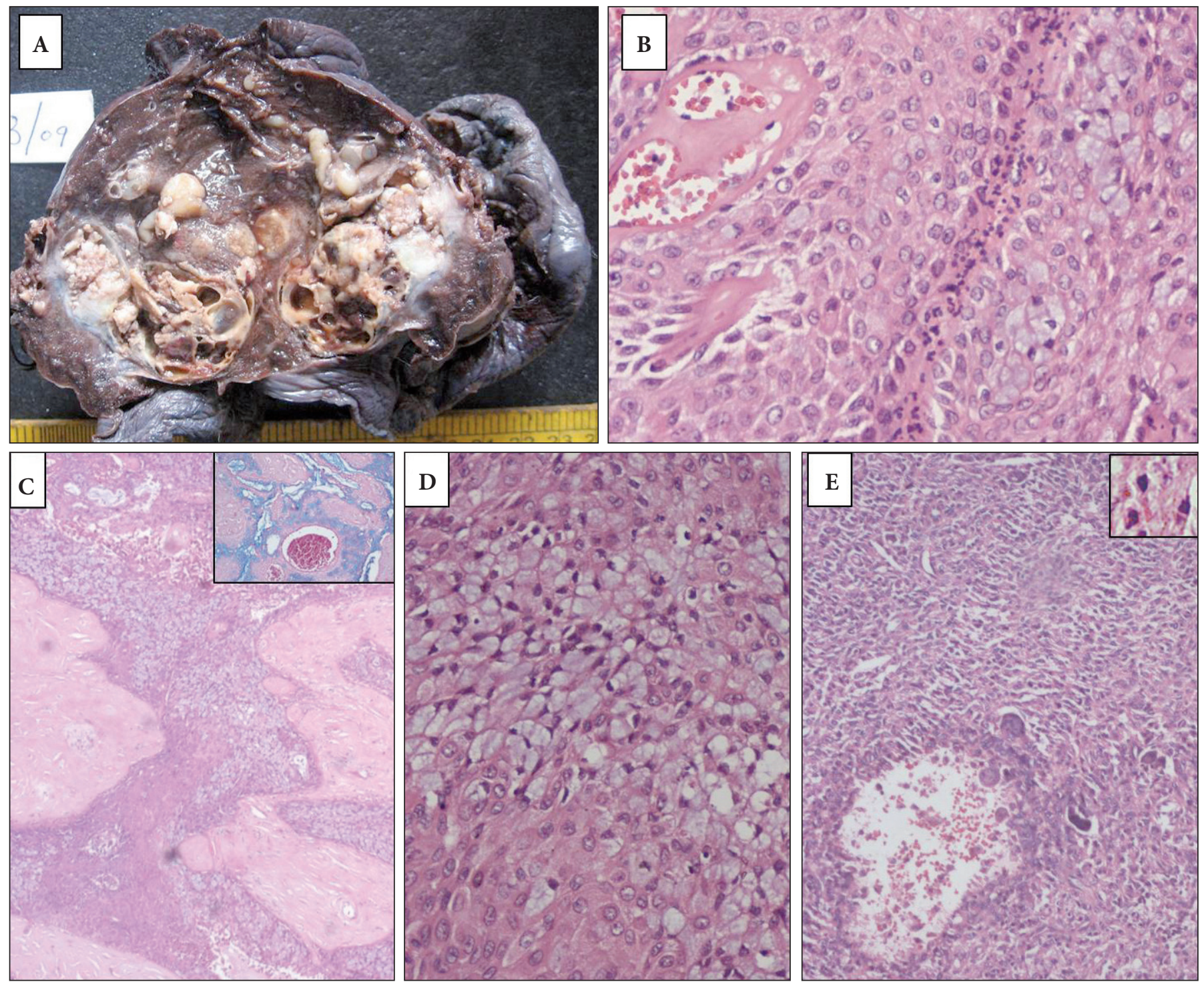

Figure 1: A) Gross specimen shows intra-bronchial mass with heterogeneous areas. H\&E stained photomicrographs show B) squamous and intermediate cells (H\&E; x200), C) mucin producing cells with Alcian blue positivity (inset), D) intermediate cells and E) sarcomatous areas with numerous giant cells, inset shows mitotic activity. 


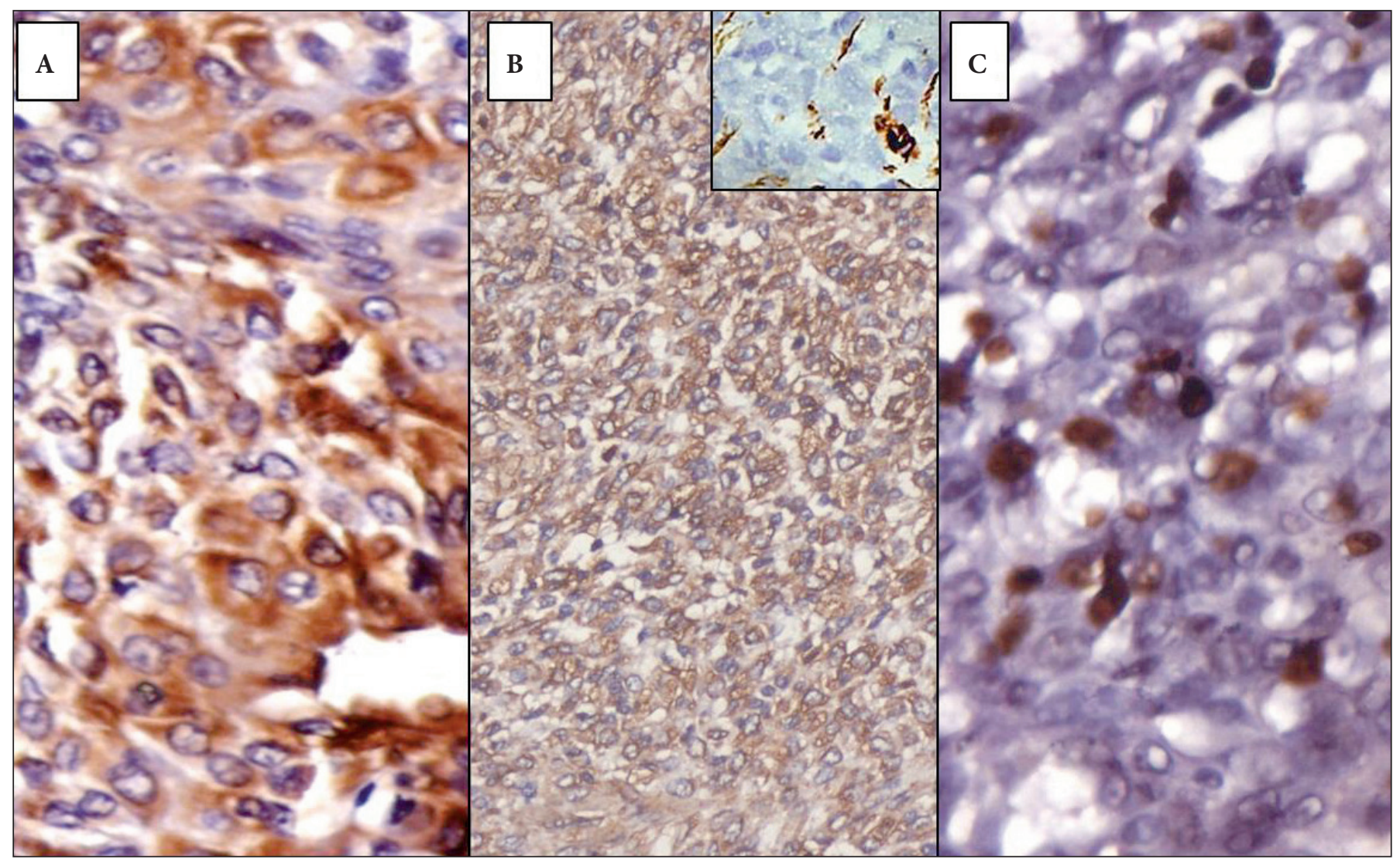

Figure 2: Immunohistochemistry with A) Pancytokeratin showing positivity in epithelial component. B) Vimentin is positive in sarcomatous component, inset shows negative immunostaining in epithelial component and C) Ki67 index of $28 \%$ indicating high proliferative capacity in neoplastic spindle cells.

Salivary gland type tumors in the lung form a distinct subset and are thought to arise from the submucosal glands of the tracheobronchial tree. The largest reported series included 62 cases. The commonest type was adenoid cystic carcinoma followed by mucoepidermoid carcinoma. Prognostically, the patients fare well when compared to conventional non-small cell lung cancers (7).

Most carcinosarcomas are seen in males (male-to-female ratio $=7: 1)$ in the sixth decade of life $(4,6)$. Associations have been reported with smoking and asbestosis (8). In a large series of pulmonary carcinosarcomas by Davis et al, $62 \%$ were endobronchial and $38 \%$ were peripheral in location (4). Interestingly, location is important as the centrally located endobronchial tumours tend to present early with cough, dyspnea or hemoptysis when compared to their peripheral counterparts. In contrast, peripheral tumors usually attain large size and present with metastasis or invade the surrounding lung. Our patient was a nonsmoker female and the tumor was endobronchial. It had expanded the lumen of the bronchus but still presented early with hemoptysis without any invasion of the pulmonary parenchyma. These findings are in accordance with those reported in literature.

The origin of carcinosarcomas is controversial. Theories of its histogenesis have been proposed by Cohen-Salmon et al. and include malignant change in a hamartoma, simultaneous epithelial and stromal malignancy, malignant change in the stroma induced by carcinoma, connective tissue metaplasia of epithelial cells and carcinomatous change in a sarcoma (9).

Squamous cell carcinoma (46\%) followed by adenocarcinoma $(31 \%)$ and adenosquamous carcinoma (19\%) account for epithelial component in most pulmonary carcinosarcomas. The sarcomatous component is usually rhabdomyosarcoma, chondrosarcoma, osteosarcoma or a combination of these (10). In most instances, the sarcoma is a poorly differentiated spindle cell sarcoma, which after extensive sampling yields differentiation. For the present case, we have undertaken extensive grossing, to the extent of exhausting the entire tumor. Multiple serial and deeper sections at various levels were studied. These did not reveal any rhabdoid, osseous or cartilaginous component. To the 
best of our knowledge this is the first case of carcinosarcoma, with a combination of mucoepidermoid carcinoma and pleomorphic undifferentiated sarcoma. Whether these patients are amenable to surgical resection or not is of little clinical significance. This is because in most of these cases the diagnosis is incomplete before surgery. Though uncommon, the tumors may metastasize; the epithelial component metastasizing to regional lymph nodes and the sarcomatous component to distant sites. Prognosis is generally poor and general rules that are applied to treatment of sarcomas must be followed (11). Survival in these patients is variable with a mean 5 year survival rate being $21.3 \%$ (7). The median survival in a series of 17 patients was 1 year (4). For all tumors less than $3 \mathrm{~cm}$ in size, and no metastasis, central location has a favorable outcome when compared to peripheral tumors $(1,4)$. At greater than $6 \mathrm{~cm}$ size, patients have reduced survival (7).

This case is reported here because of the unusual morphological combination. To the best of our literature search, it has never been earlier reported.

\section{REFERENCES}

1. Hysi I, Wattez H, Benhamed L, Porte H. Primary pulmonary myoepithelial carcinoma. Interact Cardiovasc Thorac Surg. 2011;13:226-8.

2. Haraguchi S, Fakuda Y, Sugisaki Y, Yamanaka N. Pulmonary carcinosarcoma: Immunohistochemical and ultrastructural studies. Pathol Int. 1999; 49:903-8.
3. Aurello P, Cicchini C, D’Angelo F, Mingazzini PL, Ramacciato G, De Angelis R. Squamocellular carcinoma and chondrosarcoma: A true pulmonary carcinosarcoma. Report of a case. Anticancer Res. 2003;23:3073-6.

4. Davis MP, Eagan RT, Weiland LH, Pairolero PC. Carcinosarcoma of the lung: Mayo Clinic experience and response to chemotherapy. Mayo Clin Proc. 1984;59:598-603.

5. Molina JR, Aubry MC, Lewis JE, Wampfler JA, Williams BA, Midthun DE, Yang P, Cassivi SD. Primary salivary gland-type lung cancer: Spectrum of clinical presentation, histopathologic and prognostic factors. Cancer. 2007;110:2253-9.

6. Koss MN, Hochholzer L, Frommelt RA. Carcinosarcomas of the lung: A clinicopathologic study of 66 patients. Am J Surg Pathol. 1999;23:1514-1526.

7. Nakajima $M$, Kasai T, Hashimoto $H$, Iwata $Y$, Manabe $H$. Sarcomatoid carcinoma of the lung. A clinicopathological study of 37 cases. Cancer. 1999;86:608-16.

8. Sanyal K, Sabanathan K. Lung carcinosarcoma as a rare biphasic sarcomatoid carcinoma: A case report. Cases J. 2009;2:7968.

9. Cohen-Salmon D, Michel RP, Wang NS, Eddy D, Hanson R. Pulmonary carcinosarcoma and carcinoma: Report of a case studied by electron microscopy with critical review of the literature. Ann Pathol. 1985;5:115-24.

10. Turk F, Yuncu G, Bir F, Ozturk G, Ekinci Y. Sarcomatous type sarcomatoid carcinoma of the lung with rhabdomyosarcomatous components. J Can Res Ther. 2012;8:148-50.

11. Yoshino N, Kubokura H,Yamauchi S, Ohaki Y, Koyzumi K, Shimizu K. A true pulmonary carcinosarcomas that required diagnostic differentiation from a pleomorphic adenoma: A case report. Ann Thorac Cardiovasc Surg. 2009;15:42-5. 